\title{
Interactive Terrain Simulation and Force Distribution Models in Sand Piles
}

\author{
Marta Pla-Castells ${ }^{1}$, Ignacio García-Fernández ${ }^{2}$, and Rafael. J. Martínez ${ }^{2}$ \\ 1 Previfor Simulation S.L. Alava, Spain. \\ Marta.Pla@uv.es \\ 2 LSyM. Instituto de Robótica. Universidad de Valencia \\ P.O. Box 2085, 46071 Valencia, Spain \\ \{Ignacio.Garcia, Rafael. Martinez\}@uv.es
}

\begin{abstract}
This paper presents an application of Cellular Automata in the field of dry Granular Systems modelling. While the study of granular systems is not a recent field, no efficient models exist, from a computational point of view, in classical methodologies. Some previous works showed that the use of Cellular Automata is suitable for the development of models that can be used in real time applications. This paper extends the existing Cellular Automata models in order to make them interactive. A model for the reaction to external forces and a pressure distribution model are presented and analyzed, with numerical examples and simulations.
\end{abstract}

\section{Introduction}

Granular systems dynamics has been widely studied during the last decades. The traditional approach uses fluid models and particle system models for describing the flow of granular material and the formation of heaps [1]. However, granular systems show characteristics, such as the appearance of macroscopic patterns or avalanches, that cannot be properly modelled using this approach. For this reason, Cellular Automata (CA) have been used to model and study the statistical properties of these systems [2-4].

When simulating the behaviour of a granular system in a computer graphics application, the visualization of the system's external surface and its evolution is crucial [5]. The classical models employ fluid dynamics or discrete element modelling (DEM) to study the systems. Such techniques are not appropriate, since their computational cost makes them difficult to be included in real time simulations. In contrast, CA based models are simple and describe the granular system as a grid, so they can be very efficiently rendered by graphics processors [6].

This paper deals with modelling of dry, low cohesive, granular systems for real time computer simulation of terrain manipulation (tillage, excavation, mining,... ). In this context, a simulation is considered to run in real time if computing a time interval of $\Delta t$ seconds takes less than $\Delta t$ using an standard personal computer. 


\subsection{Granular Systems Modelling}

A dry, low cohesive, granular system can be considered as a system with two layers; the standing layer, that forms the slope or the heap of the system, and the rolling layer, that is a thin layer that flows on the surface of the slope [1]. This behaviour can be modelled by means of a set of partial differential equations [8] that describe the evolution of these layers' thickness.

The model can be formulated as follows [8,9]: the system has two state variables, the height of the static layer, $s(x, y, t)$ and the height of the rolling layer, $r(x, y, t)$. The variation of these variables along time is expressed by the set of equations

$$
\begin{aligned}
& r_{t}=v \nabla(r \nabla s)-\gamma(\alpha-|\nabla s|) r \\
& s_{t}=\gamma(\alpha-|\nabla s|) r
\end{aligned}
$$

where $\alpha$ is the so called angle of repose of the system, $\gamma$ is a parameter that expresses the rate of matter transfer between layers and $v$ is the speed of the rolling layer, that is considered constant.

\subsection{Description of the CA Model}

In [7], some simplifications are taken upon this model in order to define an update rule for a CA model that reflects the behaviour of a granular system. The rolling layer is considered of constant width and the update rule is defined so that matter flows in the direction of maximum slope, indicated by the vector field $-\nabla h(x, y)$.

According to the model presented in [7], a CA on an $L \times L$ square grid is considered. This grid represents the plane on which a granular system with constant density $\rho$ is laying. The value of each cell $h(i, j) \in \mathbb{R}$ represents the height of the system on the cell's centre $\left(x_{i}, y_{j}\right)$. The set of points $\left\{\left(x_{i}, y_{j}, h(i, j)\right)\right\}_{i j}$ is a discretization of the surface $\{(x, y, h(x, y))\}$.

For each cell, $(i, j)$, an approximation to the gradient $\nabla h\left(x_{i}, y_{j}\right)$ is computed. When the slope angle obtained from this gradient $\arctan (|\nabla h|)$ is lower than the repose angle of the system $\alpha$, the value of cell $(i, j)$ remains unchanged. On the other case, if $\arctan (|\nabla h|)>\alpha$, the following update is done:

$$
\begin{aligned}
h(i, j) & \leftarrow h(i, j)-z_{+} \cdot\left(h_{x}(i, j)+h_{y}(i, j)\right) \\
h(i+1, j) & \leftarrow h(i+1, j)+z_{+} \cdot h_{x}(i, j) \\
h(i, j+1) & \leftarrow h(i, j+1)+z_{+} \cdot h_{y}(i, j)
\end{aligned}
$$

where $z_{+}$indicates the velocity of flowing matter, and $h_{x}, h_{y}$ are the partial derivatives of $h(x, y)$ respect to $x$ and $y$, computed numerically. For further detail on how the parameters of model (1) relate to the CA model, refer to [7].

The main advantages of this model are that it can be run in real time and that it can be easily managed in a $3 \mathrm{D}$ graphics environment. However, it lacks the possibility of interaction, which is a very important aspect in many virtual reality and simulation applications like a driving or civil heavy machine simulator. 
The goal of this paper is to derive from (2) some models that allow to perform an interactive simulation of a granular system. Firstly, in Sect. 2, a CA model that will be able to consider the effect of the application of vertical forces in the system will be defined. Then, in Sect. 3, a model for the computation of pressure under the granular system, based on the CA representation will be developed. Finally, in Sect. 4 a brief analysis about computational issues will be done.

\section{Interactive CA Model}

In order to provide interactivity to the model (2), a brief analysis of the stress propagation behaviour within a granular system will be done. Following, and taking into account the main properties observed, a CA model to consider the effect of applying vertical forces to the surface of the system will be proposed.

In order to maintain the constant density condition, we will assume that our system is formed by a cohesionless material, with low compressibility. Examples of such systems are dry sand, or many fertilizers formed by dry, hard particles.

\subsection{Stress Distribution in Granular Systems}

A well stated granular systems property, observed both in real systems and in simulations, is the fact that the internal stress it is not exclusively propagated vertically within the system. It also spreads horizontally, and forming some angle $\epsilon$ with the vertical line axis [10-13]. In that way, if we consider the application of a vertical force at a point $x$ on the top of the surface, the stress will be propagated not only downwards, but also horizontally pushing some material away.

In the case the force be strong enough, the pressure transmitted from point $x$ to the surroundings will make some part of the material to move up, as this is the direction where the pressure offers less resistance. After all, when the whole process ends, the result is that the height of the material at the surrounding of $x$ should have raised.

\subsection{The Model}

If we now discard any discussion about the granular system internal properties, the behaviour depicted above can be summarized as follows. If an strong enough force, $f$, is applied at a point $x$, a movement of material from point $x$ to the points surrounding it happens. This system dynamics description is analogous to the model of the granular system presented by (2), where an increase of the material in cell $i$ causes a displacement of material from that cell to its neighbours.

The new model proposed here is a modification of the original one, in which the displacement of material can be fired both, by a large difference in height, and by a big difference among the vertical forces applied to two neighbouring cells. 
Let's consider a granular system on a square plane and the CA representation defined in [7]: an $L \times L$ grid with a variable $h(i, j)$ representing the height of the system at the centre of cell $(i, j)$. Let $f(i, j)$ be the scalar value of the vertical force applied on each cell, and let $F$ be a real function $F: \mathbb{R} \rightarrow \mathbb{R}$. We define two new variables for the CA: $h_{f}$ as the composition $h_{f}(i, j):=F(f(i, j))$, and the sum $h^{\prime}:=h+h_{f}$.

For each cell $(i, j), \nabla h^{\prime}$ is computed. Then, in the cells where $\arctan \left(\left|\nabla h^{\prime}\right|\right)$ is higher than the resting angle of the system $\alpha$, the state of the automata is updated according to the following rule:

$$
\begin{aligned}
h(i, j) & \leftarrow h(i, j)-z_{+} \cdot\left(h_{x}^{\prime}(i, j)+h_{y}^{\prime}(i, j)\right) \\
h(i+1, j) & \leftarrow h(i+1, j)+z_{+} \cdot h_{x}^{\prime}(i, j) \\
h(i, j+1) & \leftarrow h(i, j+1)+z_{+} \cdot h_{y}^{\prime}(i, j)
\end{aligned}
$$

where, again, $z_{+}$represents the velocity of flowing matter, and $h_{x}^{\prime}, h_{y}^{\prime}$ are approximations to the partial derivatives of $h^{\prime}(x, y)$.

A first approximation to $F$ is to consider the height of a square column of material that weights exactly $f$. That is

$$
F(f)=\eta \frac{f}{d^{2} \rho g}
$$

where $g$ is the acceleration of gravity, $\rho$ is the density of material, $d^{2}$ is the area of a cell of the automata and $\eta>0$ is a parameter that allows to define how easily the force causes matter displacement.

The force function $F$ defined by (4) integrates very well with contact force models based on spring-damper equations. These models are very common in real-time applications and avoid large object interpenetrations by means of applying forces proportional to their overlapping $[14,15]$. In case of a collision between a rigid object and a granular system modelled by (3), the force defined by (4) will cause the granular system deformation, allowing some object advance until the deformation be large enough to eventually stop it.

\subsection{Numerical Simulations}

Figure 1 shows two examples of the use of the proposed models with this contact force computation strategy. A ball has been left fall onto two system configurations; a plane, and a heap. The pictures correspond to the final equilibrium state, for a value of $\eta=1$, with $d=0.5, \alpha=30^{\circ}$ and $z_{+}=0.05$ for the system's parameters. During the simulations, it has been observed that, as expected, if the value of $\eta$ is increased, matter flow is higher. Thus lower resistance to penetration is offered, and higher terrain deformation can be observed. This effect has not been shown in figures for space reasons.

Although the proposed model does not reproduce the inner processes that drive the interaction between a granular system and a rigid object, the effect of the interaction is the observed behaviour [16,17], extending the application range of the one presented in [7], by allowing its use in interactive real time simulations. 

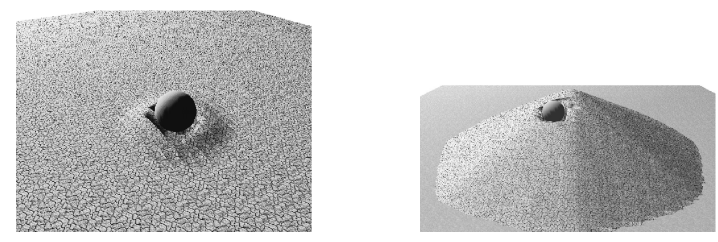

Fig. 1. A ball thrown on two different configurations of the system. The parameters of the simulation are $\eta=1$, with $d=0.5, \alpha=30^{\circ}$ and $z_{+}=0.05$.

\section{Pressure Distribution Model}

In this section, an expression to compute the pressure supported by a cell $i$ in the base of the automata, according to the state of the system, will be obtained. In order to simplify the resulting expressions, the developments will be firstly done over a unidimensional automata. Then, the way to extend the calculus to the general case will be shown.

Consider a unidimensional granular system, whose state is given by function $h(x)$, which indicates the height of every point. According to the discretization shown in [7], this system can be represented by means of a unidimensional CA, where every cell represents a point of the base. The cell value indicates the height of the system in that point.

Upon this system representation, we will split the automata in vertical slices, in such a way that the material existing over the $i$ cell will be considered as a pile of blocks of height $H$ (see Fig. 2). Let $m_{i}^{n}$ be the weight of the $n$-th block located over the $i$ cell, and let $p_{i}^{n}$ be the total pressure existing on the base of the $n$-th block situated over the $i$ cell.

In order to calculate the pressure over the base of one cell, we will consider, apart from the own block $m_{i+j}^{n}$, the pressure received by a finite number of blocks in the upper level[13], all of them centred over the $i$ cell, $\left\{p_{i+j}^{n+1}: j=-r, \ldots, r\right\}$. For simplicity we will only consider the closest blocks $\left\{m_{j}^{n+1}: j=-1,0,1\right\}$, although the development for the general case is analogous.

The way pressure of layer $n$ blocks propagates to layer $n-1$ will be expressed by means of a symmetric function $\phi: \mathbb{Z} \rightarrow[0,1]$, accomplishing

$$
\phi(k)=0 \forall k:|k|>1 ; \quad \phi(-1)+\phi(0)+\phi(1)=1
$$

so that $\phi(t)$ indicates the rate of the pressure received by the base of block $i$ in layer $n$ which is propagated to block $i+t$ in layer $n-1$.

For seek of simplicity, we will denote $\phi(i)=\phi^{i}$, and we will use the index summation convention, for which any repeated index $i$ is summed over its range, $a^{i} b_{i}:=\sum_{i} a^{i} b_{i}$. Using this notation the pressure over the base of the block at height $n$ on cell $i$ is

$$
p_{i}^{n}=m_{i}^{n}+\sum_{k=-1}^{1} \phi^{k} p_{i+k}^{n+1}=m_{i}^{n}+\phi^{k} p_{i+k}^{n+1}
$$




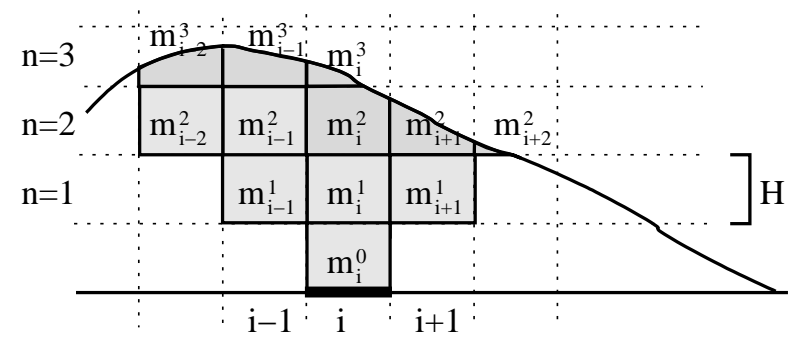

Fig. 2. Scheme of the system vertical decomposition in blocks of height $H$. The pressure that acts on the base of block $m_{i}^{n}$ depends on the weight of blocks that are at the sides above it (see text).

From this relation among the weight on a level and the weight on the immediately superior level, after recursive substitution, the total weight over a cell in the base is

$$
\begin{aligned}
p_{i}^{0}= & m_{i}^{0}+\phi^{k_{1}} m_{i+k_{1}}^{1}+\phi^{k_{1}} \phi^{k_{2}} m_{i+k_{1}+k_{2}}^{2}+\cdots \\
& +\phi^{k_{1}} \cdots \phi^{k_{n-1}} m_{i+k_{1}+\cdots k_{n-1}}^{n-1}+\phi^{k_{1}} \cdots \phi^{k_{n}} p_{i+k_{1}+\cdots k_{n}}^{n}
\end{aligned}
$$

where $l$ again takes values in $\{-1,0,1\}$. This sum ends when the top of the system is reached, since if $N \in \mathbb{N}$ is such that $N H$ overpasses the system's top, $m_{i}^{n}=0, \forall i, \forall n>N$.

Equation (7) expresses a pressure model that can be applied to a granular system represented by a CA. This expression does not depend on the update rule of the CA, but only on its state. For this reason, it can be applied both to the original CA and to the one defined in Sect. 2. Note that, when considering the action of an external force $f$, it is only necessary to add equivalent weight to the block where the force is applied.

However, (7) involves the computation of a large summation whenever the system is updated, i.e. when one of the top $m_{i}^{n}$ blocks is modified. This is unaffordable in real time applications, where a set of cells must be updated several times per second. For this reason, a rearrangement of (7) will be done to allow a more efficient pressure distribution update after a local change affecting a few cells.

Sorting the terms in (7), it can be rewritten as

$$
p_{i}^{0}=\sum_{n=0}^{N} \sum_{j=-n}^{n} a_{j n} m_{i+j}^{n}
$$

where

$$
a_{j n}=\sum_{k_{1}+\cdots+k_{n}=j} \phi^{k_{1}} \cdots \phi^{k_{n}} ; \quad a_{00}=1 .
$$

Generalization of this model to a bi-dimensional system is straightforward, from (6), and using a bi-dimensional weight expression $\phi^{i j}$. Total weight over 
$(i, j)$ cell is obtained as

$$
p_{i j}^{0}=\sum_{n=0}^{N} \sum_{s=-n}^{n} \sum_{t=-n}^{n} a_{s t n} m_{i+s j+t}^{n}
$$

where

$$
a_{s t n}=\sum_{k_{1}+\cdots+k_{n}=s} \sum_{l_{1}+\cdots+l_{n}=t} \phi^{k_{1} l_{1}} \cdots \phi^{k_{n} l_{n}} ; \quad a_{000}=1 .
$$

Therefore, from (10) and (11) it is possible to calculate pressure exerted by the system over every cell in the base.

This model depends of two parameters: the local distribution function $\phi$, and the block height, $H$. Election of function $\phi$ determines how the material is distributed from one layer to the immediately lower. As indicated before, and according to several experimental and simulation studies [12,13], load is distributed towards the sides. Thus, it is recommended to take $\phi$ as $\phi(-1)=$ $\phi(1)=\epsilon, \phi(0)=1-2 \epsilon$, with $\frac{1}{3}<\epsilon<\frac{1}{2}$. This makes that most of the load distribution will be addressed to the neighbour cells, and not to the one located just below.

By the other side, election of parameter $H$ determines the angle with respect to the vertical in which the load is propagated, $\delta=\arctan \frac{d}{H}$. This angle depends on the characteristics of each system and should be obtained experimentally. However, and according to experimental and simulation results by several authors $[11-13,19]$ most frequent values oscillate between $30^{\circ}$ and $45^{\circ}$ with respect to the vertical, which yields $d<H<2 d$.

\subsection{Numerical Simulations}

The model described previously has been implemented and simulated in order to be compared to other simulations and experimental observations. Numerical simulations have been performed, starting from an unidimensional system like the one used in the model developments. These simulations have used a system with $d=0.5, \alpha=30^{\circ}$ and $z_{+}=0.05$, with density $\rho=1$, taking $30^{\circ}, 34^{\circ}$ and $45^{\circ}$ for $\delta$, and $\phi$ with $\epsilon=0.4$. The most common experiment used in the literature has been reproduced, consisting on forming a heap by dropping material in a circular area over an horizontal plane. As results, the pressure distributions in the base of the automata, once the system reach the steady state, have been obtained.

In Fig. 3, simulation results show a curve that smoothly follows the heights of the system. This result is the same that the one obtained from other authors that have studied the case of material with constat density with numerical simulations [11], and furthermore, our results do not vary significantly from some other experimental results [10], except just in the fact that they show fluctuations that can not be viewed in our results due to the uniformity of our system.

It can be seen in Fig.3 that no substantial difference can be observed upon the election of parameter $\delta$, which indicates robustness on the procedure followed to develop the model. 

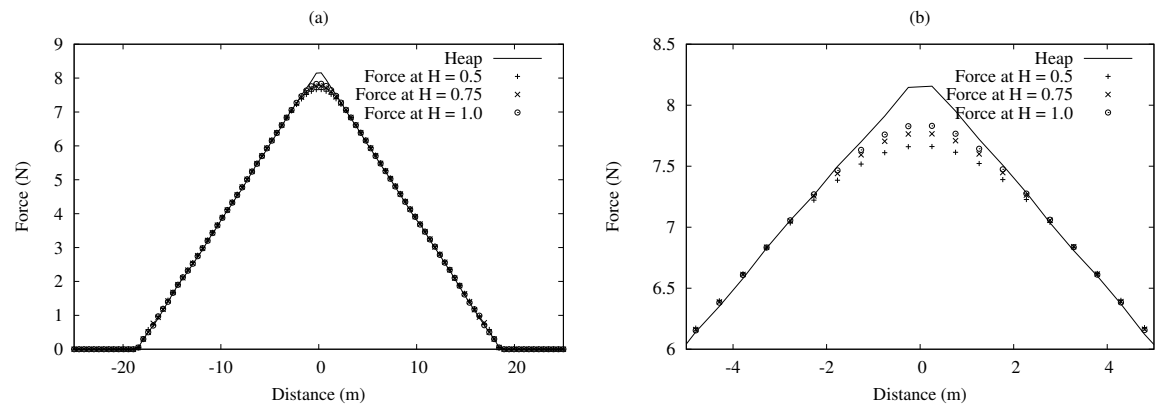

Fig. 3. Pressure distribution under a heap formed by deposition of material. The $y$ axis represents the pressure over each point of the base of the heap, that has been formed by deposition of material at point $x=0$. (a) Pressure distribution for different values of $H$. (b) Detail of the central region of the heap.

\section{Computational Cost Analysis}

In this work, the computational properties of the implemented algorithms are of great importance. The main motivation for developing CA based models has been the possibility to solve such models in the computer in real time, allowing their incorporation to graphics and simulation applications. Therefore we will focus in a detailed analysis of their computational cost, to show that they are efficient enough to make possible their inclusion in such kind of applications.

The new CA defined in this paper (3), has the same computational cost that the one described in [7]. This was predictable, since the new interactive model just incorporates four additions and two divisions per cell. Both offer a maximum cost of order $L^{2}$ for an automata $L \times L$, but an implementation sufficiently optimized offers a cost of order $L[6]$. As a result, they have been successfully used in real time graphic applications [7].

It is also necessary to evaluate the influence of the pressure model calculation in the overall performance of the new algorithm. Calculating the coefficients $a_{s t n}$ from (10) implies, if using a recursive algorithm and dynamic programming, a cost of order $N^{3}$. However, it is not necessary to make this computation every time (10) is computed, since they can be calculated only once for the chosen function $\phi$.

Once the coefficients have been calculated, pressure over a cell computation, using (10), requires $(2 n+1)^{2}$ products for every level, $n=1, \ldots, N$. However, the computation of the pressure for the whole system should be done just before the simulation starts, according to the system initial state.

As of that moment, only those cells that are modified by external causes will produce changes in the pressure distribution, affecting a square area centred in the cell. This square side will be the number of blocks of height $H$ that are 
occupied on that cell. In these cells, only those terms involving the modified block need to be calculated.

Summarizing, we can state that, by one side, the CA model described in this paper has a computational cost that, with the appropriate implementation, can be reduced to be of order one respect to the side of the automata $L$. By the other side, the pressure distribution model in the base of the $\mathrm{CA}$, although it requires a costly initialization, it can be done previously to the simulation. Once the model has been set up, the cost of the pressure update after a system modification is bounded by the square of the number of blocks of height $H$ in which the system is split, $N$. This number is usually one order of magnitude below $L$.

Therefore, the given computational costs allow the use of the automata model in the realistic simulations included in interactive real time applications.

\section{Conclusions}

In this paper, an application of CA models to interactive simulation of granular systems is presented. The main goal has been to develop efficient models of a complex system, for which the usual modelling methodologies are computationally expensive.

The proposed models complement the work previously done [7], widening it use range, and allowing $\mathrm{CA}$ as a valid alternative to classical models in granular systems simulation. They improve the previous model by adding two modes of interactivity. On the one hand, a model of the system response to an external force is proposed. On the other hand, a model for pressure distribution at the base of the system is developed.

The models have a realistic behaviour, according to granular systems bibliography. The response model of Sect. 2 behaves properly from a qualitative point of view according to the numerical tests performed, and the force distribution obtained accomplishes the main properties shown by experimentation and other simulation methodologies.

Furthermore, it has been shown that both models offer a reduced computational cost during the simulation, which makes them suitable for real time interactive applications such as computer graphics applications and simulation applications.

As a future research, some of the aspects of this work will be studied in more detail. Different expressions for the force function $f_{h}$ will be analyzed, according to several equilibrium conditions. Within this work, the pressure model has only been numerically investigated for the case $\phi(k)=0$ if $|k|>1$. Numerical experiments have to be done for the more general case. Also, some research has to be done in order to obtain revisions of the pressure model that reproduce effects observed in real and simulated systems, and that are not reproduced by the current model. In addition, new models for tool-terrain interaction will be developed in order to consider additional situations, such as the horizontal forces that appear on a vertical system-tool interface. 


\section{Acknowledgements}

This work is supported by Universitat de València, project H-TERRAIN n. 20060238. Authors would also like to thank Prof. F. Ferri for the discussion about computational cost analysis.

\section{References}

1. Aradian, A., Raphael, E., de Gennes, P. G.: Surface flows of granular materials: a short introduction to some recent models. Comptes Rendus Physique. 3 (2002) 187-196.

2. Chen, C. C., den Nijs, M.: Directed avalanche processes with underlaying interface dynamics. Physical Review E. 66 (2002).

3. Prado, C., Olami, Z.: Inertia and break of self-organized criticality in sandpile cellular-automata models. Phys. Rev. A 45 (1992) 6665-6669

4. Nerone, N., Gabbanelli, S.: Surface fluctuations and the inertia effect in sandpiles. Granular Matter. 3 (2001) 117-120

5. Müller, M., Charypar, D., Gross, M.: Procedural modeling and animation: Particlebased fluid simulation for interactive applications. ACM SIGGRAPH/Eurographics Symposium on Computer Animation (2003) 154-159

6. Pla-Castells, M.: Nuevos modelos de sistemas granulares basados en autómatas celulares para simulación en tiempo real. MSc Thesis. Escuela Técnica Superior de Ingeniería, Universidad de Valencia. (2003)

7. Pla-Castells, M., et al.: Approximation of Continuous Media Models for Granular Systems Using Cellular Automata. Lec. Not. in Comp. Sc. (2004) 230-237.

8. Bouchaud, J. P., Cates, M. E, Prakash, J. R., Edwards, S. F.: A Model for the Dynamics of Sandpile Surfaces. J. Phys. I France. 4 (1994) 1383-1410.

9. Hadeler, K. P., Kuttler, C.: Dynamical models for granular matter. Granular Matter. 2 (1999) 9-18.

10. Geng, J., Longhi, E., Behringer, R. P., Howell, D. W.: Memory in two-dimensional heap experiments. Physical Review E. 64 (2001).

11. Liffman, K., Nguyen, M., Metcalfe, G., Cleary, P.: Forces in piles of granular material: an analytic and 3D DEM study. Granular matter. 3 (2001) 165-176.

12. Snoeijer, J. H., van Hecke, M., Somfai, E., van Saarloos, W.: Force and weight distributions in granular media: Effects of contact geometry. Physical Review E. 67 (2003).

13. Snoeijer, J. H., van Hecke, M., Somfai, E., van Saarloos, W.: Packing geometry and statistics of force networks in granular media. Physical Review E. 70 (2004).

14. Stewart. D. E.: Rigid-body dynamics with friction and impact. SIAM Review, 42 (1) (2000) 3-39.

15. García-Fernández, I., Pla-Castells, M., Martínez, R. J.: New models for fast contact force computation. Industrial Simulation Conference (2003) 401-407.

16. Maciejewski J., A. Jarzebowski, A.: Experimental analysis of soil deformation below a rolling rigid cylinder. Journal of Terramechanics. 41 (2004) 223-241.

17. Fukami K., et al.: Mathematical models for soil displacement under a rigid wheel. Journal of Terramechanics. 43 (2006) 287-301.

18. Geng, J., et al. : Footprints in Sand: The Response of a Granular Material to Local Perturbations. Physical Review Letters. 87 (2001).

19. Nouguier, C., Bohatier, C., Moreau, J. J., Radjai, F.: Force fluctuations in a pushed granular material. Granular matter. 2 (2000) 171-178. 